\title{
Laparoscopic gastric resection with natural orifice specimen extraction for postulcer pyloric stenosis
}

\author{
Jan Dostalik ${ }^{1}$, Petra Gunkova ${ }^{2,3}$, Igor Gunka ${ }^{4}$, Miloslav Mazur ${ }^{2,3}$, Tomas Mrazek ${ }^{1}$ \\ ${ }^{1}$ Department of Surgery, Municipal Hospital Ostrava Fifejdy, Czech Republic \\ ${ }^{2}$ Department of Surgery, University Hospital Ostrava, Czech Republic \\ ${ }^{3}$ Department of Surgery, Faculty of Medicine, University of Ostrava, Czech Republic \\ ${ }^{4}$ Department of Surgery, University Hospital Hradec Kralove, Czech Republic
}

Videosurgery Miniinv 2014; 9 (2): 282-285

DOI: $10.5114 /$ wiitm.2014.41622

\begin{abstract}
Although natural orifice specimen extraction is now relatively widely performed, there have been no reports on gastric resection with specimen extraction through the transgastric route for peptic ulcer disease. A hybrid technique of the laparoscopic and endoscopic approach is presented in the case of a 58-year old male patient. Preoperative gastric fibroscopy showed postulcer pyloric and antral stenosis. Laparoscopic exploration confirmed gastric enlargement. Laparoscopic two-thirds gastrectomy was performed. The staple line suture of the residual stomach was excised and the specimen was extracted through the esophagus and mouth with a gastroscope. Finally, the residual stomach was closed again using linear endostaplers. Reconstruction was performed according to the Roux-en-Y method. Gastric resection using natural orifice specimen extraction (NOSE) may be a feasible operative procedure. The NOSE with the combination of standard laparoscopy and specimen extraction through a natural orifice can be considered as a bridge to natural orifice translumenal endoscopic surgery.
\end{abstract}

Key words: laparoscopy, gastric resection, natural orifice specimen extraction.

\section{Introduction}

Laparoscopic gastric surgery has well-known benefits. The advantages of this procedure in the immediate postoperative period are less pain, shorter postoperative ileus, earlier mobilization enabling faster enteral feeding, rapid recovery and excellent cosmetic results. The stress response is lower and adhesion formation is reduced. However, laparoscopically assisted techniques require the enlargement of a trocar incision, resulting in a minilaparotomy (midline, right upper quadrant) for removal of the resected specimen. The incision is also necessary to perform some steps of the operation extracorporeally. These incisions (average 6-10 cm) are much smaller than those in conventional surgery but they also present a risk of infection, wound dehiscence, pain and hernia formation, with consequent morbidity, hospital stay and increased costs [1, 2]. The incidence of surgical site infection is reported as between 0 and 11\% [3]. Hernia development occurs in less than $1 \%$ of patients and it is most common in a periumbilical midline incision [3].

Surgeons started to explore the feasibility of performing abdominal surgery endoscopically using natural orifices for extraction of the specimen and performing intracorporeal anastomosis, which means the possibility to perform totally laparoscopic surgery. Moreover, the combination of laparoscopy and natural orifice specimen extraction (NOSE) fa-

\section{Address for correspondence:}

Igor Gunka MD, PhD, University Hospital Hradec Kralove, 581 Sokolska St, 50005 Hradec Kralove, Czech Republic,

phone: +42607 679 628, e-mail: gunka@email.cz 
cilitates the execution and increases the safety of the surgical procedure because it overcomes the limitations imposed by the current surgical technologies and instrumentation. Finally, the cosmetic advantages of NOSE over the conventional procedure are notable. Developing natural orifice translumenal endoscopic surgery (NOSE) techniques may be considered as a bridge to NOTES. This progress is also possible thanks to advances in therapeutic gastrointestinal flexible endoscopy.

There have been no reports in the international literature of gastric resection using NOSE for postulcer pyloric stenosis with the transgastric route of extraction. This report describes extraction of the specimen through the esophagus and mouth in a male patient with postulcer pyloric stenosis.

\section{Case report}

A 58-year-old patient with peptic ulcer disease was investigated for nausea and abdominal fullness. Abdominal sonography was negative and subsequent gastric fibroscopy revealed postulcer and antral stenosis. Histopathology showed chronic gastritis with no signs of malignancy.

The patient's body mass index (BMI) was $26.7 \mathrm{~kg} /$ $\mathrm{m}^{2}$ and American Society of Anesthesiologists (ASA) classification was II. The patient was informed about this novel approach, associated risks, and potential complications, and signed informed consent.

Laparoscopy was performed under general anesthesia. The patient was placed in the reverse Trendelenburg position for visualization of the upper abdominal compartment, and his legs were placed in the abducted position. The operating surgeon stood between the legs and the surgical assistants stood one on each side of the patient (the first assistant on the patient's left side and the second assistant - the camera operator - on the patient's right side). Intracorporeal $\mathrm{CO}_{2}$ pressure was maintained at $12 \mathrm{~mm} \mathrm{Hg}$. Distribution of trocars was as follows: $10-\mathrm{mm}$ port for the optics in the midline $5 \mathrm{~cm}$ above the umbilicus, $12-\mathrm{mm}$ port in the right mesogastrium, 12-mm port in the left mesogastrium and $5-\mathrm{mm}$ port in the midaxillary line in the left hypochondrium. The operation commenced with a survey of the intraabdominal cavity and the next step was adhesiolysis with the harmonic scalpel. Considerable enlargement of the stomach was present. Using intraoperative endoscopy, pyloric and antral steno- sis was confirmed and the resection margin was determined. The laparoscopic grasper was used for retraction of the liver, mobilization and exposure of the stomach. Laparoscopic two-thirds gastrectomy began with mobilization of major and minor curvature of the stomach with the harmonic scalpel. The duodenum was divided using a 60- $\mathrm{mm}$ linear endostapler (ENDO GIA universal 603.5 , Covidien). The stomach was divided using two 60- $\mathrm{mm}$ linear endostaplers (ENDO GIA universal 60 3.5, Covidien). The resected stomach was transsected longitudinally and a tubule length of $30 \mathrm{~cm}$ was created. The staple line suture in the residual stomach was excised. The gastroenterologist introduced the endoscope, the specimen was captured by a polypectomy snare and was extracted through the esophagus and mouth. The residual stomach was again closed using two 60mm linear endostaplers (ENDO GIA universal 60 3.5, Covidien). Reconstruction was performed according to Roux-en-Y method using stapling technique and intracorporeal continuous suture. The sufficiency of anastomosis was confirmed by application of methylene blue solution to the nasogastric tube. The enteral feeding tube was placed behind the entero- enteroanastomosis. The abdominal cavity was flushed with antiseptic solution (povidone-iodine 1\%). The drains were placed in the subhepatic area and in the left subphrenic space.

The operation was performed under perioperative antibiotic (Ampicillin/sulbactam $3 \mathrm{~g}$ and metronidazole $1 \mathrm{~g}$ intravenously) prophylaxis and lasted $310 \mathrm{~min}$.

After surgery, the patient stayed in the intensive care unit for 10 days. The postoperative course was complicated by renal insufficiency of solitary kidney and Clostridium difficile colitis. Oral intake was initiated on postoperative day 4 , when flatus passage was documented. The enteral tube was removed on postoperative day 6 . The abdominal drains were removed on the $4^{\text {th }}$ postoperative day.

The patient was discharged to gastroenterology outpatient care on the $15^{\text {th }}$ postoperative day. Ulcer size in definitive histology was $3 \mathrm{~cm}$. Microscopic appearance confirmed chronic gastritis and fibrous tissue with no signs of malignancy (Photos 1 and 2).

\section{Discussion}

Recent innovation and progress in minimally invasive surgery has evolved to another level with 


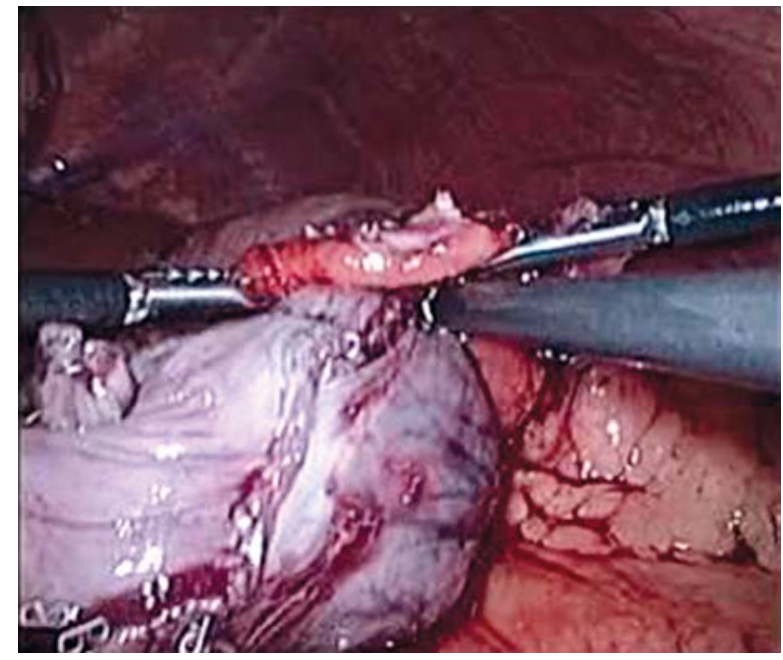

Photo 1. Insertion of the specimen into the stump of the stomach

the development of natural orifice translumenal endoscopic surgery (NOTES, surgery without scars, incisionless surgery) and laparo-endoscopic single-site surgery (LESS) in which the surgical scar is concealed within the umbilicus. The goal is to further minimize surgical trauma by eliminating the incision through the abdominal wall [4-6]. The NOSE with the combination of standard laparoscopy and specimen extraction through a natural orifice can be considered as a bridge to NOTES. The future application of NOTES for large organs in abdominal surgery depends on the improvement of endoscopes, on the invention and development of a range of new devices for flexible endosurgery instrumentation and safe and efficient closure of natural orifices [7].

There are four potential NOSE routes: transgastric [8], transrectal, transvaginal, and transurethral/ transvesical [9].

Retrieval of the colonic specimen through the anus was described for the first time by Franklin et al. [10] in 1993 using a flexible sigmoidoscope. Transrectal extraction is feasible, but carries a potential risk of infection and postoperative leakage. The risk of contamination can be minimized by thorough rectal stump washout before extraction of the specimen. Not only infectious complications but also cancer cell exfoliation, implantation and local recurrence can be eliminated by using a protective barrier or specimen bag. Understandably, this technique may not be feasible in locally advanced large and bulky tumors, in patients with anal stenosis or a small caliber rectum.

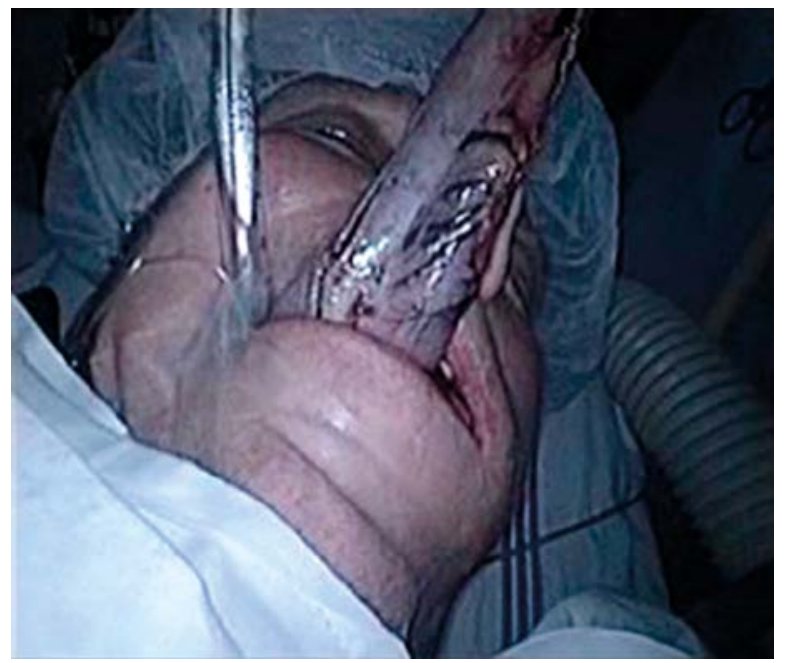

Photo 2. Extraction of the specimen

Use of the trans-vaginal route is not associated with any increased risk of postoperative leakage and infection and complications associated with incision and closure of the posterior vaginal wall are extremely rare. Transvaginal retrieval of the specimen can also avoid the potential damage of the anal sphincter and the potential risk of port-site metastasis. But the use of this route is limited to female patients. The vagina as an extraction route was first reported following laparoscopic cholecystectomy in 1993 [11] and laparoscopic nephrectomy in 2002 [12]. Yuan et al. [13] described transvaginal extraction after radical cystectomy and bilateral nephroureterectomy in 2007. Korean authors described transvaginal specimen extraction in four patients with gastric pathology (early gastric cancer) [14]. Trans-vaginal endoscopic partial gastrectomy was reported in a porcine model in 2008 [15]. Using the hybrid transvaginal minilaparoscopic-assisted natural orifice surgery (MA-NOS) in bariatric surgical treatment is also both feasible and safe [16]. According to Lacy et al. [16], with available instruments and technology, a pure nonhybrid NOTES transvaginal approach of upper abdominal organs is extremely difficult. The MA-NOS is a safe potential option to avoid abdominal incisions and related complications for the laparoscopic resection of large intra-abdominal organs.

Several studies have confirmed the technical feasibility of transgastric diagnostic and therapeutic procedures such as liver biopsy, gastrojejunostomy [17], tubal ligation [18] and cholecystectomy [19]. Some of these studies were performed on porcine 
models. Kantsevoy et al. [20] reported transgastric splenectomy on a porcine model in 2006.

Given the large volume of gastric specimens, according to some authors the use of the transgastric route for stomach specimen extraction is not feasible [14]. Our previous experience with transgastric specimen extraction and construction of the intracorporeal anastomosis allowed us to proceed to a totally laparoscopic gastric resection. This innovative technique without any minilaparotomy is also successfully used in bariatric surgery in our department, when the gastric sleeve resection specimen is removed through the transgastric route. Due to the bulkiness of the specimen after gastrectomy or subtotal gastrectomy for cancer, we consider this technique appropriate for benign diseases of the stomach. Adjustment of a bulky gastric specimen to the tubular shape prevents damage to the oesophageal wall during extraction.

\section{Conclusions}

Gastric resection using NOSE may be a feasible and alternative operative procedure for patients with postulcer stenosis. The main disadvantage of this procedure is increase in cost because of additional stapler cartridges used for repeated gastric closure and specimen tubulization. However, the combination of standard laparoscopy and specimen extraction through a natural orifice can significantly decrease wound-related complications. This combined method allows controlled implementation of NOSE techniques in clinical practice, providing a stepwise progression to the pure NOTES approach. But we must realize that NOSE procedures in gastric surgery are still in development and it is difficult to compare safety and efficacy of using these approaches with standard laparoscopic interventions.

\section{References}

1. Raymond TM, Dastur JK, Khot UP, Parker MC. Hospital stay and return to full activity following laparoscopic colorectal surgery. JSLS 2008; 12: 143-9.

2. Hackert T, Uhl W, Buchler MW. Specimen retrieval in laparoscopic colon surgery. Dig Surg 2002; 19: 502-6.

3. Palanivelu C, Rangarajan M, Jategaonkar PA, Anand NY. An innovative technique for colorectal specimen retrieval: a new era of „Natural Orifice Specimen Extraction“ (N.O.S.E.). Dis Colon Rectum 2008; 51: 1120-4.
4. Ooi BS, Quah HM, Fu CWP, Eu KW. Laparoscopic high anterior resection with natural orifice specimen extraction (NOSE) for early rectal cancer. Tech Coloproctol 2009; 13: 61-4.

5. Kurpiewski W, Pesta W, Kowalczyk M, et al. The outcomes of SILS cholecystectomy in comparison with classic four- trocar laparoscopic cholecystectomy. Videosurgery Miniinv 2012; 7: 286-93.

6. Pedziwiatr M, Matlok M, Major P, et al. Laparoscopic surgery of the spleen through single umbilical incision. Videosurgery Miniinv 2013; 8: 8-12.

7. Ryska O, Martinek J, Filipkova T, et al. Single loop- and-clips technique (KING closure) for gastrotomy closure after transgastric ovariectomy: a survival experiment. Videosurgery Miniinv 2012; 7: 233-9.

8. Kalloo AN, Singh VK, Jagannath SB, et al. Flexible transgastric peritoneoscopy: a novel approach to diagnostic and therapeutic interventions in the peritoneal cavity. Gastrointest Endosc 2004; 60: 114-7.

9. Swain P. Nephrectomy and natural orifice translumenal endoscopy (NOTES): trans-vaginal, transgastric, transrectal and transvesical approaches. J Endourol 2008; 22: 811-8.

10. Franklin ME, Ramos R, Rosenthal D, Schuessler W. Laparoscopic colonic procedures. World J Surg 1993; 17: 51-6.

11. Delvaux G, Devroey P, De Waele B, Willems G. Transvaginal removal of gallbladders with large stones after laparoscopic cholecystectomy. Surg Laparosc Endosc 1993; 3: 307-9.

12. Gill IS, Cherullo EE, Meraney AM. Vaginal extraction of the intact specimen following laparoscopic radical nephrectomy. J Urol 2002; 167: 238-41.

13. Yuan LH, Chung HJ, Chen KK. Laparoscopic radical cystectomy combined with bilateral nephroureterectomy and specimen extraction through the vagina. J Chin Med Assoc 2007; 70: 260-1.

14. Jeong SH, Lee YJ, Choi WJ, et al. Trans- vaginal specimen extraction following totally laparoscopic subtotal gastrectomy in early gastric cancer. Gastric cancer 2011; 14: 91-6.

15. Nakajima K, Takahashi T, Souma Y, et al. Transvaginal endoscopic partial gastrectomy in porcine models: the role of an extra endoscope for gastric control. Surg Endosc 2008; 22: 2733-6.

16. Lacy AM, Delgado S, Rojas OA, et al. Hybrid vaginal MA-NOS sleeve gastrectomy: technical note on the procedure in a patient. Surg Endosc 2009; 23: 1130-7.

17. Kantsevoy SV, Jagannath SB, Niiyama H. Endoscopic gastrojejunostomy with survival in porcine model. Gastrointest Endosc 2005; 62: 287-92.

18. Jagannath SB, Kantsevoy SV, Vaughn CA. Peroral transgastric endoscopic ligation of fallopian tubes with long-term survival in a porcine model. Gastrointest Endosc 2005; 61: 449-53.

19. Park PO, Bergstrom M, Ikeda K, et al. Experimental studies of transgastric gallbladder surgery: cholecystectomy and cholecystogastric anastomosis (videos). Gastrointest Endosc 2005; 61: 601-6.

20. Kantsevoy SV, Hu B, Jagannath SB, et al. Transgastric endoscopic splenectomy. It is possible? Surg Endosc 2006; 20: 522-5.

Received: 6.10.2013, accepted: 5.11.2013. 